\title{
Co-Transcriptomic Analysis by RNA Sequencing to Simultaneously Measure Regulated Gene Expression in Host and Bacterial Pathogen
}

Timothy Ravasi ${ }^{1,2 \S}$, Charalampos Mavromatis ${ }^{1,2}$, Nilesh J. Bokil ${ }^{3,4}$, Mark A. Schembri ${ }^{4,5}$ and Matthew J. Sweet ${ }^{3,4, \S}$

${ }^{1}$ Integrative Systems Biology Laboratory, Division of Biological and Environmental Sciences and Engineering, Division of Computer, Electrical and Mathematical Sciences and Engineering, King Abdullah University of Science and Technology, Thuwal, Kingdom of Saudi Arabia

${ }^{2}$ Division of Medical Genetics, Department of Medicine, University of California, San Diego, 9500 Gilman Drive, La Jolla, CA 92093 USA

${ }^{3}$ Institute for Molecular Bioscience, The University of Queensland, St Lucia, Brisbane, Qld, 4072, Australia

${ }^{4}$ Australian Infectious Diseases Research Centre, The University of Queensland, St Lucia, Brisbane, Qld, 4072, Australia

${ }^{5}$ School of Chemistry and Molecular Biosciences, The University of Queensland, Brisbane, Qld, 4072, Australia

$\S$ Co-corresponding authors:

Matthew Sweet: m.sweet@imb.uq.edu.au

Timothy Ravasi: timothy.ravasi@kaust.edu.sa

Running Head: Host-Pathogen Co-Transcriptomics 


\section{Summary}

Intramacrophage pathogens subvert antimicrobial defence pathways using various mechanisms, including the targeting of host transcriptional responses. Conversely, host defence mechanisms subject intramacrophage pathogens to stress, thus altering pathogen gene expression programs. Important biological insights can thus be gained through the analysis of gene expression changes in both the host and pathogen during an infection. Traditionally, research methods have involved the use of qPCR, microarrays and/or RNA sequencing to identify transcriptional changes in either the host or the pathogen. Here we describe the application of RNA sequencing using samples obtained from in vitro infection assays to simultaneously quantify both host and bacterial pathogen gene expression changes, as well as general approaches that can be undertaken to interpret the RNA sequencing data that is generated.

\section{Keywords:}

Host-Pathogen, Innate Immunity, Intracellular Pathogens, Macrophages, RNA sequencing, Toll-like Receptors, Transcriptomics, Urinary Tract Infections, Uropathogenic E. coli 


\section{Introduction}

Next-generation sequencing technologies have been adopted to study regulated gene expression during infection (1-7). Such methodologies enable quantification of millions of RNA transcripts in a sample, thus enabling mapping of global differences in gene expression between treatment groups $(\mathbf{8 , 9})$. Since these approaches directly determine absolute transcript levels, they overcome some of the limitations of microarray expression profiling (10,5). Most previous infection model studies employing RNA

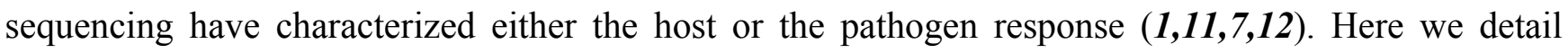
RNA sequencing and analysis methodology that we have used to successfully evaluate the expression profiles of two organisms from the same sample (Figure 1).

The model system that we have employed for host-pathogen co-transcriptomics relates to urinary tract infections (UTI), one of the most common infections in humans. Uropathogenic Escherichia coli (UPEC), the main causative agent of UTIs, can invade and replicate within bladder epithelial cells (13), and some UPEC strains can also survive within macrophages (14). To further understand UPECmacrophage interactions, we recently performed co-transcriptomics of mouse bone morrow-derived macrophages (BMM) challenged over a $24 \mathrm{~h}$ time course with the UPEC genome sequenced reference strains, UTI89 (cystitis strain) and 83972 (asymptomatic bacteriuria strain). These strains possess contrasting phenotypes for intramacrophage survival. In this chapter, we use our mouse macrophageUPEC infection studies as a model system to present an approach for performing in vitro infection assays, isolating and sequencing total RNA from co-cultures, extracting sequence data for each organism studied, and analyzing the simultaneous changes in expression that occur in the interacting organisms. These methods could be adapted for the study of other host-pathogen systems, as well as the detailed analysis of organisms that interact in symbiotic relationships. 


\section{Materials}

\subsection{BMM Infection assays}

1. Specific pathogen free C57BL/6 male mice at 6 to 8 weeks age.

2. Disposable plasticware and general reagents: $10 \mathrm{~mL}$ syringe and $21 \mathrm{G}$ needle; $18 \mathrm{G}$ blunt needle; 100 mm square bacteriological petri dishes (Thermo Scientific); Luria Bertani (LB) medium; LB agar plates (no antibiotic selection); phosphate buffered saline; 24 well tissue culture (TC) plates; $50 \mathrm{~mL}$ TC tubes.

3. BMM media with antibiotic (complete media) - RPMI1640 containing 10\% heat-inactivated fetal bovine serum, $50 \mathrm{U} / \mathrm{mL}$ penicillin (Life Technologies), $50 \mu \mathrm{g} / \mathrm{mL}$ streptomycin (Life Technologies), 2 mM L-glutamine (GlutaMAX, Life Technologies).

4. Antibiotic-free BMM media (media above, but without penicillin and streptomycin).

5. Gentamicin solution (TC grade).

6. Glycerol stocks of uropathogenic E. coli strains UTI89 (15) and 83972 (16).

\subsection{RNA Preparation, Sequencing and Analysis}

1. Disposable plasticware for RNA preparation.

2. RNeasy Maxi kits (Qiagen).

3. Ambion MICROBEnrich kit (Life Technologies).

4. Ribo-Zero rRNA removal kit (Epicenter).

5. Agilent RNA 6000 Nano Kit (Agilent Technologies).

6. Qubit ${ }^{\circledR}$ RNA BR Assay Kit (Life Technologies).

7. TruSeq ${ }^{\circledR}$ Stranded mRNA Sample Preparation Kit (Illumina).

8. SuperScript II Reverse Transcriptase (Invitrogen). 
9. Agencourt AMPure XP 60ml kit (Beckman Coulter).

10. TruSeq PE Cluster Kit v3 (Illumina).

11. TruSeq SBS v3-HS kit (Illumina). 


\section{Methods}

\subsection{Infection Assays of BMM for Determining Intracellular Survival}

All solutions should be aliquoted into $50 \mathrm{~mL}$ tubes to prevent contamination of stocks. Quality control infection assays should be performed in parallel with the infection assays to be used for RNA generation, using the same preparations of cells and bacteria. This ensures that matched intramacrophage survival and expression profiling data are generated.

1. On day 0 , sacrifice mice, collect femurs and tibias and place the bones in complete media.

2. Remove muscle and surrounding tissue from the femoral and tibial bones, then under sterile conditions clean the bones with 70\% ethanol and place in complete media (see Note 1).

3. Remove the very tips of the femurs and tibias using surgical scissors, and flush the bone cavity with complete media and a $21 \mathrm{G}$ needle into a $50 \mathrm{~mL}$ tube to collect bone marrow (BM) cells (see Note 2).

4. Wash BM cells twice with complete media (centrifuge at $500 \mathrm{~g}$ for 5 min each time).

5. Divide BM cells into four $100 \mathrm{~mm}$ square petri dishes with $15 \mathrm{~mL}$ complete media per plate, and culture in the presence of $10,000 \mathrm{U} / \mathrm{mL}$ recombinant colony stimulating factor-1 (CSF-1), as described previously $(17,18)$.

6. On day 5, add fresh complete media $(5 \mathrm{~mL})$, plus fresh CSF-1 to final concentration of $10,000 \mathrm{U} / \mathrm{mL}$ (see Note 3).

7. Also on day 5, streak an antibiotic-free LB agar plate of specific UPEC strain of interest from glycerol stock under aseptic conditions (see Note 4).

8. On day 6, harvest BMM. To do so, discard the media from the $100 \mathrm{~mm}$ square petri dishes, then wash the plates twice with magnesium- and calcium-free PBS, transferring this wash to $50 \mathrm{~mL}$ tubes. Add $10 \mathrm{~mL}$ sterile PBS to the petri dishes and leave for $5 \mathrm{~min}$. Using a $10 \mathrm{~mL}$ syringe and $18 \mathrm{G}$ blunt 
needle, wash the BMMs off the plate and collect in the same $50 \mathrm{~mL}$ tubes. Spin the cells down at $500 \mathrm{~g}$ for $5 \mathrm{~min}$ and resuspend in antibiotic-free BMM media. Plate BMM out in antibiotic-free BMM media in 24 well TC plates: 2 wells for each treatment and 2 wells for control. Plate a total of 200,000 cells/well in a total volume of $900 \mu \mathrm{L}$ with CSF-1 (see Note 5).

9. On day 6, also set up overnight bacterial cultures. UPEC strains are cultured statically in LB broth at $37^{\circ} \mathrm{C}$ overnight in conical flasks. Static culture is employed to enrich for the expression of type 1 fimbriae.

10. On day 7, spin down overnight cultures of bacteria at $4000 \mathrm{~g}$ for $10 \mathrm{~min}$. Wash two times with PBS, then resuspend in $10 \mathrm{~mL}$ PBS.

11. Assess expression of type 1 fimbriae on the surface of UPEC isolates using the yeast agglutination test (19), prior to performing infection assays. In short, mix a drop of $10 \%$ yeast solution with a bacterial suspension on a glass slide and observe for agglutination. Strains expressing type 1 fimbriae will agglutinate (see Note 6).

12. Using a spectrophotometer, measure absorbance at $600 \mathrm{~nm}$ of the bacterial suspension. Adjust the $\mathrm{A}_{600 \mathrm{~nm}}$ of the bacterial suspension to $0.7 \mathrm{AU}$. This is equivalent to $1 \times 10^{8} \mathrm{cfu} / \mathrm{mL}$ (see Note 7).

13. Dilute bacteria to the required concentration in antibiotic-free media such that the volume of bacterial suspension to be added to macrophage cultures is $100 \mu \mathrm{L} /$ well. For example, for a multiplicity of infection (MOI) of 10 , dilute to $2 \times 10^{7} \mathrm{cfu} / \mathrm{mL}: 100 \mu \mathrm{L}$ is thus $2 \times 10^{6} \mathrm{cfu}$, which will be used to infect 200,000 BMM (see Note 7).

14. Plate appropriately diluted bacterial suspension on antibiotic-free LB agar plates and incubate overnight at $37^{\circ} \mathrm{C}$ for MOI determination (see Note 7).

15. Add $100 \mu \mathrm{L}$ bacterial suspension per well of 24 well plate of BMM, and incubate for $1 \mathrm{~h}$ at $37^{\circ} \mathrm{C}$.

16. Aspirate the supernatant from wells, wash twice with $1 \mathrm{~mL}$ antibiotic-free media containing 200 $\mu \mathrm{g} / \mathrm{mL}$ gentamicin, add another $1 \mathrm{~mL}$ media containing gentamicin $(200 \mu \mathrm{g} / \mathrm{mL})$ to the wells and incubate at $37^{\circ} \mathrm{C}$ for $1 \mathrm{~h}$ (see Note 8 and $\left.\mathbf{9}\right)$. 
17. Aspirate supernatant from wells, wash wells twice with $1 \mathrm{ml}$ antibiotic-free media, then add $1 \mathrm{~mL}$ antibiotic-free media containing $20 \mu \mathrm{g} / \mathrm{mL}$ gentamicin to the wells and incubate at $37^{\circ} \mathrm{C}$ for the appropriate time (see Note $\mathbf{8}$ and $\mathbf{1 0}$ ).

18. To assess intracellular bacterial loads at designated time points, aspirate media, wash twice with 1 $\mathrm{mL}$ antibiotic-free media, add $1 \mathrm{~mL} \mathrm{PBS} / 0.01 \%$ Triton $\mathrm{X}-100$ to each well, leave for $5 \mathrm{~min}$ at room temperature, then pipette up and down to ensure complete lysis before harvesting lysate into a $1.5 \mathrm{~mL}$ eppendorf tube.

19. Vortex the eppendorf tube and plate out $50 \mu \mathrm{L}$ on LB agar plates at multiple dilutions. Incubate overnight.

20. Count colonies and calculate intracellular bacterial numbers (see Note $\mathbf{1 1}$ and 12).

\subsection{Infection Assays of BMM for RNA Preparation}

To assess bacterial and mammalian RNA expression profiles by RNA sequencing, infection assays need to be scaled up. Thus, infection assays are carried out essentially as described above, but in a 10 $\mathrm{cm}$ round TC plate (Figure 2).

1. Plate out $7 \times 10^{6} \mathrm{BMM}$ in $10 \mathrm{~mL}$ antibiotic-free media one day prior to infection.

2. Infect with bacteria and perform washes as described in section 3.1.

3. After incubation for designated times (e.g. 2, 4, 24 h), wash cells with ice-cold saline, then lyse on plates with the appropriate lysis buffer, as per the specific RNA purification protocol.

4. To determine a basal level of bacterial gene expression, culture bacteria statically in $10 \mathrm{~mL}$ antibiotic-free BMM media for $2 \mathrm{~h}$, using the same inoculum that is used to infect the BMM. At the end of $2 \mathrm{~h}$, spin down the bacteria $(4000 \mathrm{~g}$ for $10 \mathrm{~min}$ ) and lyse with appropriate lysis buffer. Total 
RNA should also be prepared from uninfected BMM at the same time points as used for co-infections (Figure 2).

5. Extract RNA using RNeasy Maxi kits (Qiagen, Hilden, Germany). Add $3.5 \mathrm{~mL}$ RLT buffer (with added 2-mercaptoethanol) per $10 \mathrm{~cm}$ dish, as well as to bacterial pellets from bacteria grown separately.

7. Scrape cell lysates off the plate using a cell scraper for maximum yield. Homogenize lysates by passing them 20 times through a $25 \mathrm{G}$ needle and extract total RNA, as per the manufacturer's protocol (including off-column DNase digestion). For enrichment of microbial RNA in co-culture samples, use Ambion MICROBEnrich (Life Technologies).

8. rRNA should be removed from all purified RNA samples using kits, such as Ribo-Zero (Epicenter), targeting mammalian and Gram-negative bacterial rRNAs.

9. Prior to sequencing, all RNA preparations should be quantified and assessed for protein and reagent contamination using Qubit ${ }^{\circledR}$. RNA samples for analysis must be selected based on a spectroscopic $\mathrm{A}_{260} / \mathrm{A}_{280} \mathrm{~nm}$ ratio of $1.8-2.0$, and an $\mathrm{A}_{260} / \mathrm{A}_{230} \mathrm{~nm}$ ratio of greater than 1.5 (see Note 13).

\subsection{RNA Sequencing, Alignment, Annotation and Differential Gene Expression Analyses}

Next-generation sequencing analyses for $n \geq 3$ biological replicates can be performed on an Illumina Cluster Station and the Illumina HiSeq 2000 System. The first step is to convert the mRNA within the total RNA into a library of template molecules of known strand origin using the reagents provided in the Illumina ${ }^{\circledR}$ TruSeq ${ }^{\circledR}$ Stranded mRNA Sample Preparation Kits. The library generated is suitable for subsequent cluster generation with the TruSeq PE Cluster Kit. The cluster is now ready for sequencing using the TruSeq SBS v3-HS kit that determines the DNA sequence of each cluster on a flow cell using sequencing by synthesis technology on the HiSeq sequencing systems.

1. Use the Solexa Automated Pipeline for image analysis, base calling and quality calibration. 
2. Quality control of RNA-Seq reads can be preprocessed by custom java script, which checks the raw sequence data from high-throughput sequencing pipelines for any problems. RNASeq reads can be subjected to quality trimming in order to remove Illumina adapters, low quality leading and trailing bases, bases with low average quality, and reads shorter than 36 bases long. Trimmomatic, a flexible read trimming tool for Illumina NGS data (20), can be used for this purpose.

3. To align reads, bowtie indexes should be created for the host and pathogen reference genomes using the bowtie-build algorithm and reference sequences from the GenBank database. We recommend TopHat to map the raw RNA-Seq reads (fastq files) to the reference genomes. TopHat uses Bowtie as an alignment engine and breaks up reads that Bowtie cannot align on its own into smaller pieces (21). Using the standard parameters, both reads of paired-end libraries can be mapped. All simulations should be performed using a high-memory node cluster system.

4. The approach for transcription annotation is dependent upon the number of replicates per condition. If the number of replicates per condition is large $(n>10)$, after running TopHat, we suggest providing the resulting alignment files to Cufflinks to generate a transcriptome assembly for each condition. During this analysis step, adapter tags, mitochondrial sequences, poly A, poly C, and phiX sequences, and remaining ribosomal sequences will be filtered out. Estimated normalized expression levels will be reported in fragments (i.e. reads) per kilobase of exon per million mapped reads (FPKM). These assemblies will be compared with annotation files using the Cuffcompare utility, which is included in the Cufflinks package $(22,23)$. On the other hand, if the number of replicates per condition is small $(n<$ 10), after running TopHat, we suggest providing the resulting mapped short reads in SAM format, along with the gene annotation in GTF format, to GFOLD count to count the number of reads mapped to each gene (24). Estimated normalized expression levels will be reported in reads per kilobase of exon per million mapped reads (RPKM). 
5. The number of replicates also affects the approach to be used for differential gene expression analysis. Where the number of replicates is large, the reads and assemblies can be further imported to Cuffdiff, which calculates expression levels and tests the statistical significance of observed changes. For comparison of differentially-expressed genes across samples, the number of raw clean tags in each library is normalized to FPKM using the Cufflinks package. A threshold can be set for the minimum number of alignments in a locus needed to test for statistically significant changes in that locus between samples. If no testing is performed, changes in the locus will be deemed insignificant, and the changes observed in the locus will not contribute to corrections for multiple testing. The Cuffdiff output files can then be imported to cummeRbund, which plots abundance and differential expression results as commonly used expression plots for quality control (23). Where the number of replicates is small, the reads can be further imported to GFOLD diff, which generalizes the fold change by considering the posterior distribution of log fold change, such that each gene is assigned a reliable fold change (see Note 14). The GFOLD output files can be further processed for quality control using the R package.

\subsection{Ortholog Identification, Dimensionality Reduction, Clustering and Pathway Analysis}

1. Identification of orthologous genes is an essential component of studies comparing multiple host and/or pathogen species. We have used the Ensembl database (25) for this purpose (see Note 15). All genes that share orthology should be converted to orthologous groups (OG). In the case of one-to-one orthology, an OG will contain a single gene from each species. In the cases of one-to-many and manyto-many orthology relationships, an OG is defined as the set of genes that share this relationship. A new annotation file should be prepared with only orthologous interactions prior to performing a second differential expression analysis, this time comparing species (A vs B vs C etc) for each time point. This step is necessary since it eliminates genes present in one species but not the other. 
2. Dimensionality reduction enables exploration of the relationships between conditions in RNA sequence data sets. The minimum classification error (MCE) method performs a nonlinear dimension reduction by embedding high-dimensional data points into a lower-dimensional space using the minimum curvilinear kernel in combination with multidimensional scaling (MDS) (26), or alternatively, the singular value decomposition (SVD) (27). The nonlinear data distances for MDS or SVD are computed and stored in the minimum curvilinear kernel as the traversal distances over the minimum spanning tree between the data points (sample conditions) in the multidimensional space (the gene space). The minimum spanning tree is constructed from the Pearson correlation-based distances between the samples (26):

correlation_based_distance $(x, y)=1-$ Pearson_Correlation $\left(\right.$ Sample $_{x}$, Sample $\left._{y}\right)$

MCE is a parameter-free projection algorithm that is particularly effective in discriminating classes in small- $n$ (samples: here conditions), large- $m$ (features: here gene expression) datasets using only the first dimension of embedding (26). In the UPEC/macrophage RNA sequence datasets, the fact that $n<<m$ led us to adopt the MCE algorithm for unsupervised analysis of the patterns present between the different sample conditions.

3. Genes with similar expression patterns often serve overlapping functions or co-exist within a biological pathway. Accordingly, the optimal number of clusters in the dataset can be determined by performing a cluster analysis of gene expression patterns using the R package NbClust. Selected lists of divergently expressed genes can be further compiled for hypothesis testing and clustering using the Ward's methodology. Significantly enriched gene ontologies (GOs) and pathways can be identified by mapping all divergently expressed genes to terms in the GO and KEGG databases by applying twosided Fisher's exact and $\chi^{2}$ tests, respectively $(\mathbf{2 8 , 2 9})$. P-values should be corrected by calculating the FDR, and only GOs and pathways with a FDR $<0.01$ should be chosen. 
4. Analysis of promoter sequences of divergently expressed genes can identify likely transcription factors (TFs) and upstream signaling pathways mediating changes in gene expression. Promoter sequences can be retrieved using regulatory sequence analysis tools (RSAT), with subsequent input into the RSAT matrix-scan tool along with host-related matrices for transcription factor binding site (TFBS) prediction (30-32). The RSAT output should be filtered using an adjusted P-value $<0.05$ as a cut-off, and lists of the most significant TFBSs and their known corresponding TFs should be compiled. The expression profiles of the host divergently expressed genes can be clustered, and each cluster can be correlated with the TF profiles using Pearson correlation in R. Finally, the clusters can be further annotated using GO to gain insights into the molecular processes in which each TF is involved. 


\section{Notes}

1. While cleaning femurs and tibias with ethanol do not leave the bones in ethanol for more than $3-5$ min, as this can cause the bones to go brittle.

2. While flushing femurs and tibias do not re-aspirate flushed media using the needle. This can result in cell shearing and decreased yield.

3. Since BMM consume CSF-1, cell density is an important variable if the CSF-1 concentration becomes limiting. We therefore use a high concentration of CSF-1 and replenish the CSF-1 during differentiation to avoid variability between different cell preparations. The actual CSF-1 concentration to be used (in $\mathrm{U} / \mathrm{mL}$ or $\mathrm{ng} / \mathrm{mL}$ ) will vary, depending on the source.

4. There is no selection, be careful with your technique.

5. Whether to include CSF-1 in BMM cultures post-differentiation is a point of contention. We normally include CSF-1 in cultures used for our experiments, because this growth factor is constitutively present in vivo. Furthermore, macrophages do proliferate locally in various tissue environments. However, in experiments where cell proliferation may confound data interpretation, CSF-1 should be excluded. In any case, whether this growth factor is included in experimental conditions should be clearly stated, given that it has major effects on the functions of mature macrophages.

6. Optimal type 1 fimbriae expression should result in a rapid time to agglutination (i.e. less than 5 seconds). If this is not achieved, a second round of static culture, where the inoculum is obtained by removing $100 \mu \mathrm{L}$ from the air-liquid interface of the first culture, should be performed.

7. As soon as possible after determining cfu/mL, use bacterial suspension for infection assays and plate the inoculum out for MOI determination (to avoid further bacterial growth, which will alter the MOI that is actually used). If this is not possible, place the inoculum at $4^{\circ} \mathrm{C}$ or on ice until the plating can be 
performed. Note that each laboratory should independently determine the correlation between $\mathrm{A}_{600 \mathrm{~nm}}$ and $\mathrm{cfu} / \mathrm{mL}$ for determining the MOI.

8. There are several washing steps during infection assays, which can result in detachment of BMM from the tissue culture plastic surface. To avoid this, add the wash solution slowly along the side of the well or plate.

9. There is some literature to suggest that gentamicin can affect intracellular bacteria $(33,34)$. If comparing intramacrophage survival and/or responses of different bacterial species or strains, ensure that there are no differences in their sensitivity to gentamicin, as this may confound data interpretation.

10. To confirm that the gentamicin wash has killed all extracellular bacteria, the supernatant from the final wash with antibiotic-free media can be plated on antibiotic-free LB agar.

11. To calculate intracellular bacterial numbers, plate out at least 2 dilutions per sample i.e. neat and 1/50 or 1/100 etc. The dilution depends on how high your MOI is, the length of the infection assay and the specific pathogen being investigated. Calculation of MOI and intracellular bacterial numbers:

$\mathrm{cfu} / \mathrm{mL}=$ No. of colonies $\mathrm{x}$ dilution factor $\mathrm{x}$ 1000/volume of plated solution.

12. Plate out the supernatant and the lysate from the uninfected samples. This will serve as a control to confirm there was no contamination during the course of the infection assay.

13. Extensive quality control is crucial when undertaking large-scale analyses such as RNA sequencing. Prior to performing RNA sequencing, qPCR analyses should be performed for specific host and pathogen genes for which biological effects are already known.

14. GFOLD overcomes the shortcoming of $p$-value by measuring relative expression changes instead of the significance of whether a gene is differentially expressed under different conditions (24). It also overcomes the shortcoming of using fold change, which suffers from the fact that the fold changes of genes with low-read counts are not as reliable as those of genes with high read counts. 
15. There are many freely available resources that can be used to determine orthology relationships. Each method will result in some differences in orthology predictions based on the database used and enrichment score cut-off.

\section{Acknowledgments}

This work was supported by grants from the National Health and Medical Research Council (NHMRC) of Australia (APP1005315 and APP1068593). M.J.S. is supported by an Australian Research Council Future Fellowship (FT100100657), as well as an honorary NHMRC Senior Research Fellowship (APP1003470). M.A.S. is supported by an ARC Future Fellowship (FT100100662). T.R. and C.H.M. are supported by The King Abdullah University of Science and Technology. 


\section{References}

1. Hegedus, Z., Zakrzewska, A., Agoston, V.C., et al. (2009) Deep sequencing of the zebrafish transcriptome response to mycobacterium infection. Mol Immunol 46, 2918-2930.

2. Huang, Q., Dong, S., Fang, C., et al. (2012) Deep sequencing-based transcriptome profiling analysis of Oryzias melastigma exposed to PFOS. Aquat Toxicol 120-121, 54-58.

3. Jager, D., Sharma, C.M., Thomsen, J., et al. (2009) Deep sequencing analysis of the Methanosarcina mazei Gol transcriptome in response to nitrogen availability. Proc Natl Acad Sci U S A 106, 21878-21882.

4. Nie, Q., Sandford, E.E., Zhang, X., et al. (2012) Deep sequencing-based transcriptome analysis of chicken spleen in response to avian pathogenic Escherichia coli (APEC) infection. PLoS One 7, e41645.

5. t Hoen, P.A., Ariyurek, Y., Thygesen, H.H., et al. (2008) Deep sequencing-based expression analysis shows major advances in robustness, resolution and inter-lab portability over five microarray platforms. Nucleic Acids Res 36, e141.

6. Wang, F., Hu, S., Liu, W., et al. (2011) Deep-sequencing analysis of the mouse transcriptome response to infection with Brucella melitensis strains of differing virulence. PLoS One 6, e28485.

7. Xiang, L.X., He, D., Dong, W.R., et al. (2010) Deep sequencing-based transcriptome profiling analysis of bacteria-challenged Lateolabrax japonicus reveals insight into the immune-relevant genes in marine fish. BMC Genomics 11, 472.

8. Morozova, O. and Marra, M.A. (2008) Applications of next-generation sequencing technologies in functional genomics. Genomics 92, 255-264.

9. Wang, Z., Gerstein, M. and Snyder, M. (2009) RNA-Seq: a revolutionary tool for transcriptomics. Nat Rev Genet 10, 57-63.

10. Llorens, F., Hummel, M., Pastor, X., et al. (2011) Multiple platform assessment of the EGF dependent transcriptome by microarray and deep tag sequencing analysis. BMC Genomics 12, 326.

11. Ordas, A., Hegedus, Z., Henkel, C.V., et al. (2011) Deep sequencing of the innate immune transcriptomic response of zebrafish embryos to Salmonella infection. Fish Shellfish Immunol 31, 716-724.

12. Xiao, S., Jia, J., Mo, D., et al. (2010) Understanding PRRSV infection in porcine lung based on genome-wide transcriptome response identified by deep sequencing. PLoS One 5, e11377.

13. Hannan, T.J., Totsika, M., Mansfield, K.J., et al. (2012) Host-pathogen checkpoints and population bottlenecks in persistent and intracellular uropathogenic Escherichia coli bladder infection. FEMS Microbiol Rev 36, 616-648.

14. Bokil, N.J., Totsika, M., Carey, A.J., et al. (2011) Intramacrophage survival of uropathogenic Escherichia coli: differences between diverse clinical isolates and between mouse and human macrophages. Immunobiology 216, 1164-1171.

15. Mulvey, M.A., Schilling, J.D. and Hultgren, S.J. (2001) Establishment of a persistent Escherichia coli reservoir during the acute phase of a bladder infection. Infect Immun 69, 45724579 .

16. Andersson, P., Engberg, I., Lidin-Janson, G., et al. (1991) Persistence of Escherichia coli bacteriuria is not determined by bacterial adherence. Infect Immun 59, 2915-2921.

17. Hume, D.A. and Gordon, S. (1983) Optimal conditions for proliferation of bone marrowderived mouse macrophages in culture: the roles of CSF-1, serum, Ca2+, and adherence. J Cell Physiol 117, 189-194. 
18. Tushinski, R.J. and Stanley, E.R. (1983) The regulation of macrophage protein turnover by a colony stimulating factor (CSF-1). J Cell Physiol 116, 67-75.

19. Schembri, M.A., Hasman, H. and Klemm, P. (2000) Expression and purification of the mannose recognition domain of the FimH adhesin. FEMS Microbiol Lett 188, 147-151.

20. Bolger, A.M., Lohse, M. and Usadel, B. (2014) Trimmomatic: a flexible trimmer for Illumina sequence data. Bioinformatics 30, 2114-2120.

21. Kim, D. and Salzberg, S.L. (2011) TopHat-Fusion: an algorithm for discovery of novel fusion transcripts. Genome Biol 12, R72.

22. Roberts, A., Trapnell, C., Donaghey, J., et al. (2011) Improving RNA-Seq expression estimates by correcting for fragment bias. Genome Biol 12, R22.

23. Trapnell, C., Roberts, A., Goff, L., et al. (2012) Differential gene and transcript expression analysis of RNA-seq experiments with TopHat and Cufflinks. Nat Protoc 7, 562-578.

24. Feng, J., Meyer, C.A., Wang, Q., et al. (2012) GFOLD: a generalized fold change for ranking differentially expressed genes from RNA-seq data. Bioinformatics 28, 2782-2788.

25. Flicek, P., Amode, M.R., Barrell, D., et al. (2014) Ensembl 2014. Nucleic Acids Res 42, D749755.

26. Cannistraci, C.V., Ravasi, T., Montevecchi, F.M., et al. (2010) Nonlinear dimension reduction and clustering by Minimum Curvilinearity unfold neuropathic pain and tissue embryological classes. Bioinformatics 26, i531-539.

27. Cannistraci, C.V., Alanis-Lobato, G. and Ravasi, T. (2013) Minimum curvilinearity to enhance topological prediction of protein interactions by network embedding. Bioinformatics 29, i199209.

28. Huang da, W., Sherman, B.T. and Lempicki, R.A. (2009) Bioinformatics enrichment tools: paths toward the comprehensive functional analysis of large gene lists. Nucleic Acids Res 37, 113.

29. Huang da, W., Sherman, B.T. and Lempicki, R.A. (2009) Systematic and integrative analysis of large gene lists using DAVID bioinformatics resources. Nat Protoc 4, 44-57.

30. Thomas-Chollier, M., Defrance, M., Medina-Rivera, A., et al. (2011) RSAT 2011: regulatory sequence analysis tools. Nucleic Acids Res 39, W86-91.

31. Thomas-Chollier, M., Sand, O., Turatsinze, J.V., et al. (2008) RSAT: regulatory sequence analysis tools. Nucleic Acids Res 36, W119-127.

32. Turatsinze, J.V., Thomas-Chollier, M., Defrance, M., et al. (2008) Using RSAT to scan genome sequences for transcription factor binding sites and cis-regulatory modules. Nat Protoc $3,1578-1588$.

33. Drevets, D.A., Canono, B.P., Leenen, P.J., et al. (1994) Gentamicin kills intracellular Listeria monocytogenes. Infect Immun 62, 2222-2228.

34. Hamrick, T.S., Diaz, A.H., Havell, E.A., et al. (2003) Influence of extracellular bactericidal agents on bacteria within macrophages. Infect Immun 71, 1016-1019. 


\section{Figure Legends}

Figure 1. Bioinformatic analysis pipeline. Summary of the four stages of analysis (RNA Preparation and Sequencing; Data Pre-Processing; Alignment and Differential Expression Analysis; Functional Analysis), as well as details of the steps involved in each stage.

Figure 2. Experimental design of BMM infection assays, used for quantification of intracellular bacterial loads and RNA generation. 


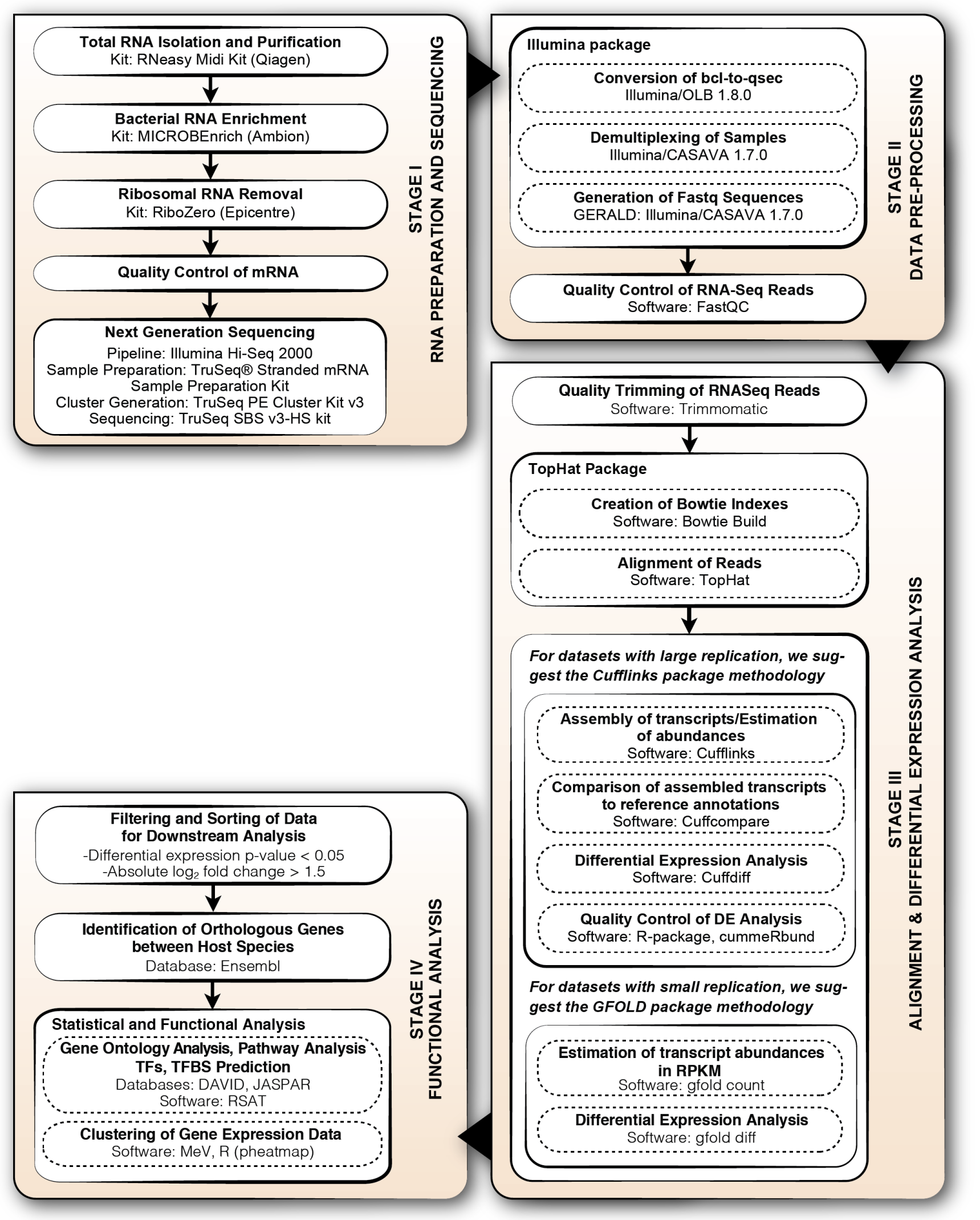

Figure 1 


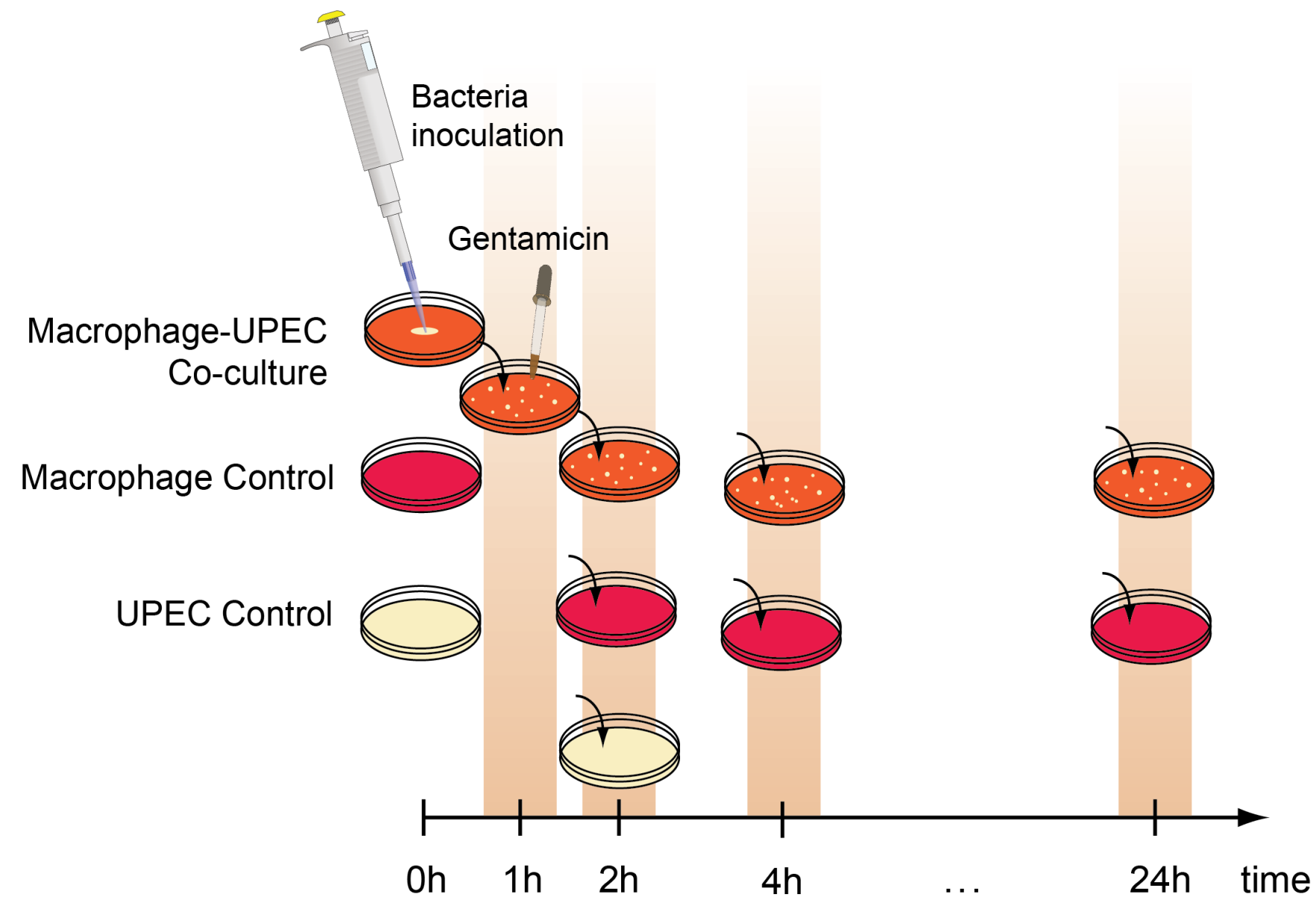

Figure 2 